\title{
Circumstellar Disks in Very Young Embedded Clusters
}

\author{
Naibí Mariñas ${ }^{1}$, Elizabeth A. Lada ${ }^{1}$, Paula S. Teixeira ${ }^{2}$ \\ and Charles J. Lada ${ }^{3}$ \\ ${ }^{1}$ Astronomy Department, University of Florida,Gainesville, FL 32611 \\ ${ }^{2}$ University of Vienna, Tuerkenschanzstrasse 17, 1180, Vienna, Austria \\ ${ }^{3}$ Harvard-Smithsonian CFA, Cambridge, MA
}

\begin{abstract}
We used FLAMINGOS near-IR photometry and spectroscopy and Spitzer mid-IR photometry to study disk fractions in the 1 to 2 Myr old NGC2264 clusters. We find that stars with masses $<0.3$ solar masses have lower disk fractions than stars of solar mass or higher at these early ages. We also find that most disks disappear within the first $4 \mathrm{Myr}$, which is consistent with previous studies of disk lifetimes. Our study suggests that either some very low mass stars form without disks or that their disks are less massive and/or colder than predicted from models and not detected with Spitzer/Flamingos sensitivities.
\end{abstract}

Keywords. stars: pre-main-sequence, stars: circumstellar matter

\section{Introduction}

The standard model of star formation predicts that all stars are formed by accretion and are surrounded by circumstellar disks. Planets will form in these disks and this process is deeply connected to the amount of gas and dust available. However, do all stars really begin their lives surrounded by disks?

Near- and mid-IR studies of the frequency distribution of disks in young stellar clusters show that disks dissipate quickly, within 3 to 5 Myr, (Haisch, Lada \& Lada 2001; Hernandez et al. 2008) and that their evolution seems to depend on the mass of the central star, intermediate mass stars have shorter disk lifetimes than solar and low mass stars (e.g. Haisch, Lada \& Lada 2000; Carpenter et al. 2006; Lada et al. 2006). These short disk lifetimes imply that even when we consider clusters with young ages, between 3 to $5 \mathrm{Myr}$ old, this mass dependent disk evolution already dominates the observed disk fractions. It is the stellar population with ages $<2 \mathrm{Myr}$ that offers a window to investigate the initial frequency distribution of disks.

To address this issue, we have recently investigated the disk fractions of the young (1 to $2 \mathrm{Myr}$ ) population of the NGC2264 northern and southern clusters (Marinas et al. 2013, Marinas et al. in preparation).

\section{Overview}

We used FLAMINGOS near-IR spectra (R 1000 and $\mathrm{S} / \mathrm{N}>50)$ to obtain spectral types of cluster members by comparing the spectral features and continuum shape of the stars with those of standards. For sources later than M2, the broad $\mathrm{H}$ band absorption feature was used, for K3-M2 stars we used line ratios of $\mathrm{MgI}(1.50 \mu \mathrm{m})$ and $\mathrm{SiI}(1.59 \mu \mathrm{m})$ to $\mathrm{OH}(1.69 \mu \mathrm{m})$, which are sensitive to effective temperatures. For the earlier sources, HI $(1.68 \mu \mathrm{m})$ was used as well. Using this spectral classification and JHK FLAMINGOS photometry we estimated luminosities for all the sources. Spectral types were converted 
to temperature by using the temperature scale of Kenyon and Hartmann (1995) and Luhman et al. (2003). Placing the sources on the H-R diagram, and using theoretical evolutionary pre-main-sequence (PMS) tracks (Baraffe et al. 1998, Chabrier et al. 2000, Siess et al. 2000) we derived median ages of 1.4 Myr for the NGC2264 southern cluster and 1.8 Myr for the NGC2264 northern cluster. Given the uncertainties associated with theoretical PMS model ages, the two clusters seem to be coeval.

To obtain infrared excess for the sources we compared the expected SED slope from the photosphere alone over the wavelength range 1.68 to $8 \mu \mathrm{m}$, estimated by using NextGen models given each star effective temperature and surface gravity, with the slope of the SED from measured fluxes corrected for interstellar extinction. The excess for each source was correlated with stellar mass and age. A more detailed description of the data reduction and analysis can be found in Marinas et al. 2013.

The final sample included 226 stars from the two NGC2264 clusters.

\section{Results}

Disk Lifetimes Infrared excess in this sample decreases rapidly with age. While the disk fraction in stars younger than $2 \mathrm{Myr}$ is $56 \pm 4 \%$, the disk fraction for stars 2 to $4 \mathrm{Myr}$ is $40 \pm 5 \%$ and the disk fraction for stars older than 4 Myr is only $24 \pm 6 \%$.

Initial Disk Fractions and Stellar Mass Disk fractions in the young NGC2264 increase with stellar mass, resembling the disk distributions found in lower stellar density regions like ChaI and Taurus (Luhman et al. 2010). We find a disk fraction of $68 \pm 8 \%$ for 1 to 3 solar mass stars, a disk fraction of $51 \pm 5 \%$ for 0.3 to 1 solar mass stars and a disk fraction of $42 \pm 7 \%$ for 0.1 to 0.3 solar mass stars. Since we expect stars in very young clusters and those in low density environments to be less evolved, this result suggest that stellar mass might play a role in the initial distribution of disks.

Disk Evolution and Stellar Mass In the 3 Myr old cluster IC348, disk fraction peaks for the very low mass stars at about $50 \%$, and decreases for the more massive stars (Luhman et al. 2010). This is the opposite of what we find in NGC2264. The difference in the disk fraction dependance on stellar mass between these two clusters of similar stellar densities, but different ages, could be the result of mass dependent disk evolution. While a very young region like NGC2264 would still be more representative of the original disk distribution, a faster disk evolution for more massive stars would deplete disks around these higher mass stars faster and result in the distribution found in the older IC348.

Studies of other nearby clusters of similar ages are needed to investigate the role of stellar mass in the formation and early retention of circumstellar disks.

\section{References}

Baraffe, I., Chabrier, G., Allard, F., \& Hauschildt, P. H. 1998, A\& A, 337, 403

Carpenter, J. M., Mamajek, E. E., Hillenbrand, L. A. et al., 2006, ApJL, 651, 49

Chabrier, G., Baraffe, I., Allard, F., \& Hauschildt, P. 2000, ApJ, 542, 464

Haisch, K. E. Jr, Lada, E. A., \& Lada, C. J. 2000, AJ, 120, 1396

Haisch, K. E. Jr., Lada, E. A., \& Lada, C. J. 2001, AJ, 121, 2065

Hernandez, J., Hartmann, L., Calvet, N. et al., 2008, ApJ, 686, 119

Kenyon, S., J. \& Hartmann, L. 1995, ApJS, 101, 117

Lada, C. J., Muench, A. A., Luhman, K. L. et al., 2006, AJ, 131,1574

Luhman, K. L, Stauffer, J. R., Muench, A. A., Rieke, G. H. et al., 2003, ApJL, 593, 1093

Luhman, K. L., Allen, P. R., Espaillat, C., Hartmann, L., \& Calvet, N. 2010, ApJS, 186, 111

Marinas, N., Lada, E. A., Teixeira, P. S., \& Lada, C. J. 2013, ApJ, 772

Siess L., Dufour, E., \& Forestini, M. 2000, A\& $A, 358,593$ 